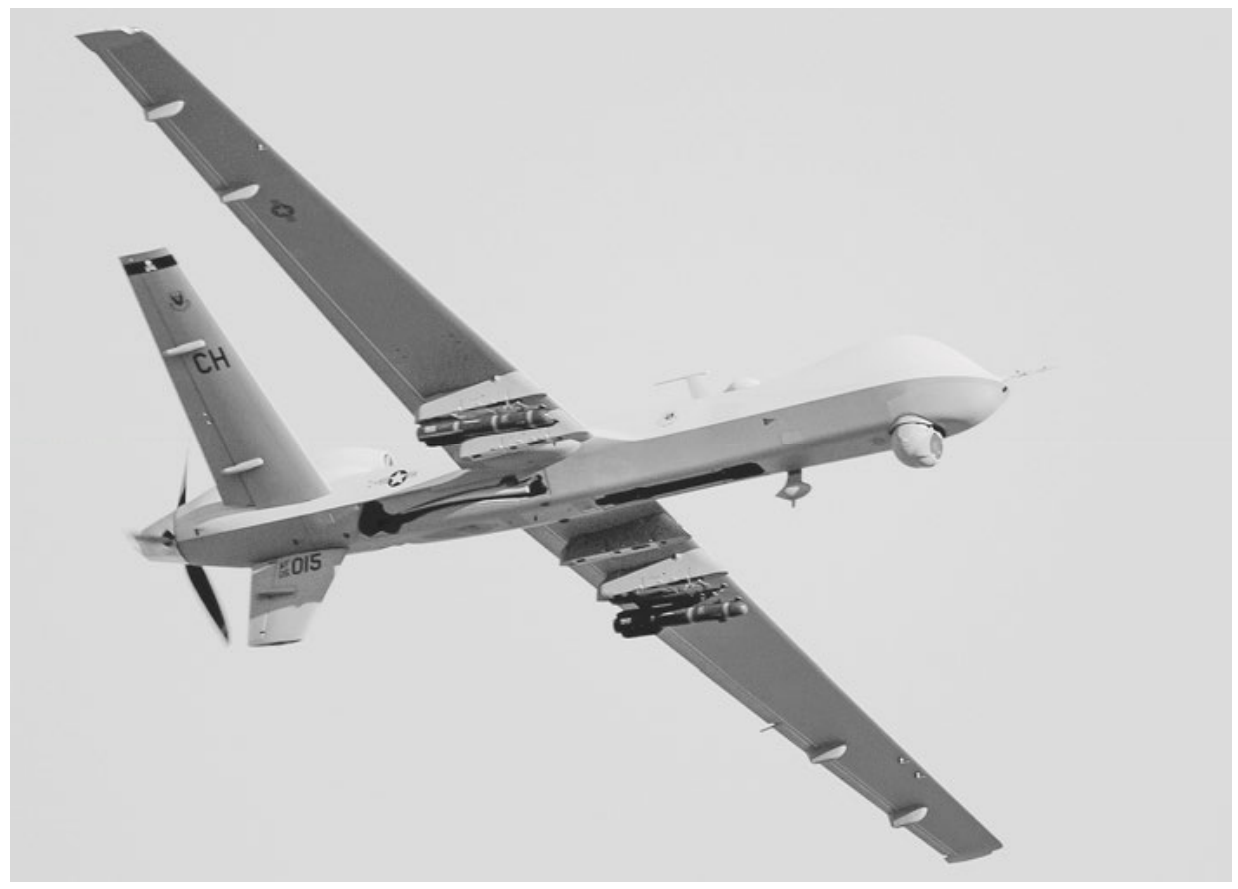

Abb. 1: MQ-9 Reaper-Drohne: Fotografie für die US Air Force von Master Sgt. Robert W. Valenca vom 10. November 2007. 


\section{Drohnen und Helden}

\section{Ulrich Bröckling}

Am 4. Februar 2002 feuerte eine Drohne vom Typ Predator eine Hellfire-Rakete auf drei Männer in der Nähe der afghanischen Stadt Khost und tötete sie. Man vermutete, die CIA habe einen der drei, wegen seiner Körpergröße und seiner grauen Haare, für Osama bin Laden gehalten. Ein offensichtlicher Irrtum, wie sich bald herausstellte. Ein Pentagon-Sprecher erklärte im Nachhinein, „[w]e're convinced that it was an appropriate target“, musste jedoch einräumen, „[w]e do not know yet exactly who it was". ${ }^{1}$ Journalisten berichteten später, bei den Getöteten habe es sich um Zivilisten gehandelt, die auf dem Gelände eines verlassenen Mudjaheddin-Camps nach Altmetall suchten.

Bei dieser Tötungsaktion handelte sich um die erste bekannt gewordene Operation einer bewaffneten Drohne. Zu Aufklärungszwecken waren die Predators schon seit 1994 eingesetzt worden, mit einem Waffensystem hatte man sie allerdings erst kurz zuvor ausgerüstet. In der Testphase hatten Experten befürchtet, der rückwärtige Feuerstrahl der Raketen könne die Leichtfluggeräte zerstören. Das geschah nicht, und damit begann der rasante Aufstieg der Remotely Piloted Aircraft beziehungsweise Unmanned Combat Air Vehicles (UCAVs), so die offizielle Bezeichnung.

Die Bush-Administration setzte in der Folge bewaffnete Drohnen in Afghanistan und Pakistan zunächst zur Tötung sogenannter ,High-Value-Targets ${ }^{\star}$ ein, die Angriffe richteten sich gegen bekannte Talibanführer oder Mitglieder von Al Qaida. Unter Obama wurde das Programm massiv ausgebaut, allein während seiner ersten Amtszeit zählte man fünf Mal so viele Angriffe wie in den acht Jahren der Bush-Präsidentschaft. Inzwischen machen Drohnen ein Drittel der USamerikanischen Kriegsluftflotte aus. ${ }^{2}$ Die US-Regierung betreibt zwei Drohnenprogramme: ein militärisches, das feindliche Kräfte in den Kriegsgebieten in Afghanistan und dem Irak bekämpft, und ein geheimes unter Verantwortung der CIA, das sich gegen Terrorverdächtige in der gesamten Welt richtet und auch in Gebieten operiert, in denen keine US-Truppen stationiert sind. ${ }^{3}$ Dokumentiert sind verdeckte Drohnenangriffe vor allem im Jemen, in Somalia und Syrien. Die Obama-Regierung weitete indes nicht nur die Einsatzgebiete, sondern auch die Ziele der Angriffe aus. Neben der Tötung namentlich bekannter Terrorverdächtiger, die auf einer vom Präsidenten unterzeichneten Todesliste aufgeführt sind, setzt sie

1 John Sifton: A Brief History of Drones, in: The Nation, 27. Februar 2012.

2 Asawin Suebsaeng: Drones. Everything You Ever Wanted to Know But Were Always Afraid to Ask, in: Mother Jones, 5. März 2013.

3 Jane Mayer: The Predator War. What are the risks of the C.I.A.'s covert drone program?, in: The New Yorker, 26. Oktober 2009. 
auf sogenannte signature strikes. Diese richten sich gegen „groups of men who bear certain signatures, or defining characteristics associated with terrorist activity “ ${ }^{4}$ Die Identität der Zielpersonen ist zunächst noch unbekannt, signiert` werden sie aufgrund ihres Verhaltens. Anhand einer Lebensmusteranalyse (pattern of life analysis) werden persönliche Profile angelegt, die sich aus den von den Überwachungskameras der Drohnen gesammelten Fakten, aber auch aus anderen Daten, beispielsweise aus der Auswertung von Mobilfunkverbindungen speisen. In der Summe ergibt das Profiling ein Gesamtbild der zeitlichen, räumlichen und sozialen Verhaltensparameter eines Menschen. Auf diese Weise wird das Töten sukzessive automatisiert; Algorithmen entscheiden, wer sterben muss. ${ }^{5}$ Welche Merkmale die Zielpersonen im Einzelnen als Verdächtige ausweisen, das bleibt geheim. Zivile Opfer werden kurzerhand wegdefiniert: Nachdem John Brennan, Obamas Berater in Sachen Terrorbekämpfung, 2011 stolz verkündet hatte, die Technik sei inzwischen so weit fortgeschritten, dass es im Jahr zuvor so gut wie keinen kollateralen Todesfall gegeben habe, deckte die „New York Times“ auf, dass die amtlichen Dokumente alle Männer im wehrfähigen Alter, die sich im Gebiet des Drohneneinsatzes aufhalten, pauschal als Kombattanten einstuften. Korrigiert wurde dies, sofern explizite Hinweise auf die Unschuld der Getöteten auftauchten, allenfalls postum. ${ }^{6}$ In Regierungskreisen kursierte ein Scherz, nach dem die CIA schon überzeugt sei, ein Trainingscamp für Terroristen gefunden zu haben, wenn Drohnenkameras drei Männer entdeckt hätten, die Freiluftgymnastik betrieben. ${ }^{7}$

Recherchen unabhängiger Journalisten belegen demgegenüber einen hohen Anteil getöteter Zivilisten; ihr Anteil bewegt sich zwischen 12 und 35 Prozent. Allein für Pakistan gehen sie - Stand Anfang Mai 2015 - von 423 bis 962 zivilen Drohnenopfern aus, darunter zwischen 172 und 207 getötete Kinder, bei einer Gesamtzahl der Getöteten zwischen 2449 und 3949. ${ }^{8}$ Rechtlich gesehen ist die Politik der gezielten Tötungen höchst umstritten: Selbst Juristen, die solche Aktionen im Rahmen bewaffneter zwischenstaatlicher Konflikte durch das Völkerrecht gedeckt sehen, stufen Drohnenangriffe auf dem Gebiet von Staaten, mit denen man sich nicht im Kriegszustand befindet, als völkerrechtswidrig ein.

4 Daniel Klaidman: Kill or Capture. The War on Terror and the Soul of the Obama Presidency, New York 2012, S. 41.

5 Vgl. Nils Markwardt: Drohnenkrieg. Überwachen und vernichten, in: Die Zeit, 27. Oktober 2014.

6 „Mr. Obama embraced a disputed method for counting civilian casualties that did little to box him in. It in effect counts all military-age males in a strike zone as combatants, according to several administration officials, unless there is explicit intelligence posthumously proving them innocent", Jo Becker / Scott Shane: Secret 'Kill List' Proves a Test of Obama's Principles and Will, in: New York Times, 29. Mai 2012.

7 Carsten Luther: Amerikas Drohnenkrieg bleibt Geheimaktion, in: Die Zeit, 4. Januar 2013.

8 Das Bureau of Investigative Journalism in London dokumentiert die Zahl der Toten und Verletzten seit 2004: http://www.thebureauinvestigates.com/category/projects/drones/dronesgraphs/, 2. Mai 2015. 
Das Skandalon der präemptiven Tötung Verdächtiger ohne Anklage und Gerichtsurteil, die mit dem zynischen Euphemismus eines Kollateralschadens belegten Opfer unter der Zivilbevölkerung, die Traumatisierung der gesamten Bevölkerung in den betroffenen Regionen, die täglich 24 Stunden die Drohnen über sich kreisen hören und sehen und die jederzeit fürchten müssen, ohne Vorwarnung unter Raketenbeschuss zu geraten - all das ist nicht Gegenstand der folgenden Überlegungen. ${ }^{9}$ Im Rahmen einer Auseinandersetzung mit ,Objekten des Heroischen' soll vielmehr gefragt werden, wie die Unmanned Combat Air Vehicles traditionelle Vorstellungen militärischen Heldentums erodieren lassen, beziehungsweise welche Bedeutung die Erosion heroischer Orientierungen in zeitgenössischen westlichen Gesellschaften für den rasanten Siegeszug dieser Waffensysteme besitzt. Anders ausgedrückt: Es geht um Drohnen als paradigmatische Objekte eines vermeintlich postheroischen Zeitalters. Ausgeblendet bleiben damit ebenfalls der militärische Einsatz von Drohnen zu Aufklärungszwecken, wie auch zivile Nutzungen dieser Technologien etwa zur Kartierung von Waldgebieten oder archäologischen Ausgrabungsstätten, zur Inspektion von Brücken und Pipelines, zum Transport von Medikamenten und Laborproben - oder als Spielzeug für Spanner. ${ }^{10}$

Geführt wird der Drohnenkrieg von US-amerikanischer Seite derzeit vor allem mit einer Weiterentwicklung der Predator-Drohne, die unter dem Namen MQ-9 Reaper - was auf Deutsch sowohl Mähmaschine als auch Sensenmann bedeutet firmiert und für ,Hunt and kills-Operationen ausgelegt ist. Mit einer Länge von elf und einer Flügelspannweite von zwanzig Metern kann diese Drohne bis zu dreißig Stunden in der Luft bleiben; sie fliegt in einer Höhe von bis zu 15.000 Metern und deckt dabei einen Einsatzradius von mehr als 3000 Kilometern ab. Bestückt ist sie zum einen mit Hellfire-Luft-Boden-Raketen und lasergesteuerten Präzisionsbomben, zum anderen mit einem Aufklärungssystem, das zahlreiche Infrarot- und Videokameras sowie Richtlaser kombiniert, bis zu 65 Streaming-Bilder gleichzeitig an unterschiedliche Adressaten sendet und es ermöglicht, eine Fläche von vier mal vier Kilometern in hoher Bildauflösung aus unterschiedlichen Blickwinkeln zu überwachen. Aus einer Flughöhe von 3,2 Kilometern lassen sich damit Nummernschilder von Autos entziffern. Das System trägt den mythologischen Namen Gorgon Stare, Gorgonenblick. Das noch in der Planung befindliche Nachfolgesystem heißt Argus IS, nach dem allsehenden Riesen aus der griechischen Mytho-

9 Vgl. dazu die ausgezeichnete, von Wissenschaftlern der Stanford University und der New York University gemeinsam herausgegebene Dokumentation: International Human Rights and Conflict Resolution Clinic at Stanford Law School and Global Justice Clinic at NYU School of Law: Living Under Drones. Death, Injury and Trauma to Civilians from US Drone Practices in Pakistan, Sept. 2012, http://www.livingunderdrones.org/wp-content/ uploads/2013/10/Stanford-NYU-Living-Under-Drones.pdf, 2. Mai 2015.

10 Vgl. Daniel Schulz / Johannes Gernert: Das fliegende Auge, in: Badische Zeitung, 3. Januar 2015; Julia Littmann: „Drohnen“ überm Damenbad: Wurden Besucherinnen fotografiert?, in: Badische Zeitung, 22. August 2015; EB: Bluttransport. Im Notfall schneller per Drohne, in: Deutsches Ärzteblatt 112, Heft 4, 23. Januar 2015, B 128. 
logie, der auch panóptes genannt wird. Panoptisch ist das System in der Tat. Die unklassifizierten Videos von Drohnenangriffen, die man bei YouTube anschauen kann, geben nur einen vagen Eindruck über die Genauigkeit der Bilder und Daten, die den Militärs und der CIA zur Verfügung stehen.

Neben einem Bodenteam, das für Start und Landung der Drohne zuständig ist, sind drei Leute für ihren Einsatz erforderlich. Diese Crew besteht aus einem Piloten, der das System fernsteuert, einem Sensor Operator, der die verschiedenen Kameras, Radargeräte und Sensoren bedient, und einem Mission Intelligence Coordinator, der die Kommunikation mit Analysten, Datenbanken und anderen Crews übernimmt. ${ }^{11}$ Während das Bodenteam auf einem Flughafen in regionaler Nähe zum Einsatzgebiet stationiert ist, sitzen die Operatoren im Schichtdienst auf einer Tausende von Kilometern entfernten Militärbasis in Nevada oder im Pfälzerwald vor ihren Bildschirmen. Die Daten werden ihnen in Echtzeit per Satellit übermittelt. Die räumliche Distanz geht allerdings einher mit einer virtuellen Nähe: Mit dem ferngesteuerten Super-Zoom verfolgen die Drohnen-Operatoren ihre Zielpersonen über Tage, Wochen, manchmal Monate rund um die Uhr. Sie registrieren, wann diese das Haus verlassen, wohin sie gehen, mit wem sie sich treffen. So entsteht eine einseitige, aber geradezu intime soziale Beziehung. Und wenn sie die Hellfires abgefeuert haben, sehen sie aus ebenso großer Nähe, was diese anrichten: Tod und Zerstörung in einem Umkreis von mindestens fünfzehn Metern. Anders als Bomberpiloten, die nach einem Abwurf weiterfliegen und den Schrecken, den sie bringen, niemals zu Gesicht bekommen, bleibt das elektronische Auge nach dem Treffer weiterhin auf den Punkt gerichtet, an dem die Opfer vernichtet wurden.

Es ist diese Virtualität des Tele-Kriegs, es ist der geographische Abstand zwischen waffenbewehrtem Flugobjekt und Bedienungspersonal und damit verbunden die Diskrepanz zwischen der tödlichen Gewalt, denen die Opfer der Drohnenangriffe ausgesetzt sind, und der Sicherheit der Crews in ihren Operation Rooms, welche diese Form der Kriegführung anstößig erscheinen lässt. Kritik kommt nicht zuletzt von militärischer Seite: Der Drohnenkrieg sei ein „,virtueless war', requiring neither courage nor heroism“, zitiert ein Artikel im „New Yorker“ den vormaligen British Air Chief Marshall Sir Brian Burridge: „There’s something about pilotless drones that doesn't strike me as an honorable way of warfare. As a classics major, I have a classical sense of what it means to be a warrior", erklärt ein ehemaliger Army Ranger im selben Beitrag. ${ }^{12}$ Ein 19-jähriger Drohnenpilot berichtet von seinem ersten Angriff, bei dem er Fahrer und Beifahrer eines mit Maschinengewehr bestückten Pickups tötete, die eine Patrouille amerikanischer Bodentruppen in Südafghanistan beschossen: „You feel bad. You don't feel worthy. I'm sitting here

11 Peter M. Asaro: The Labor of Surveillance and Bureaucratized Killing: New Subjectivities of Military Drone Operators, in: Social Semiotics 23, Heft 2, 2013, S. 196-224.

12 Mayer: Predator War (Anm. 3). 
safe and sound, and those guys down there are in the thick of it, and I can have more impact than they can. It's almost like I don't feel like I deserve to be safe." 13

Die Strategie des gezielten Tötens widerspricht dem soldatischen Ethos mit seiner Idee eines ,gerechten Kampfes'. Demnach gilt es als unehrenhaft, einen Feind anzugreifen und zu töten, ohne sich derselben Gefahr auszusetzen. Zum Kriegshelden kann nur werden, wer auch zum Selbstopfer bereit ist. Der Drohnenkrieg bricht mit dieser elementaren Reziprozität, was jedoch keineswegs ein neues Phänomen darstellt. ${ }^{14}$ Die Einwände gegen Distanzwaffen sind vielmehr so alt wie diese: Bereits in der „Ilias“ beschimpft Diomedes den „Mädchenbeäugler“ Paris als „nichtsgeachteten Weichling“, nachdem ihn dieser, versteckt hinter einer Säule, mit einem Pfeil verletzt hatte: „Wenn du mit offner Gewalt in Rüstungen wider mich kämest", wettert Diomedes, selbst ein Heros wie alle Krieger des homerischen Epos, „[w]enig frommte dir wohl dein Geschoß und die häufigen Pfeile“. ${ }^{15}$ Nicht anders als in der Antike impliziert das Verdikt der Feigheit auch heute eine sexuelle Depotenzierung. So hat die offizielle Bezeichnung für die ferngesteuerten Waffensysteme - Unmanned Combat Air Vehicles - einen deheroisierenden, weil die Männlichkeit anzweifelnden Doppelsinn: ,Unmanned` bedeutet im Englischen nicht nur unbemannt, sondern auch entmannt. ${ }^{16}$

Der Vorwurf, Distanzwaffen seien die Waffen der Feiglinge, bindet ex negativo militärisches Heldentum an das Vorbild des Kampfes Mann gegen Mann. In dieser „Negation des Technischen bei gleichzeitiger Apologie des Zweikampfs“17 treffen sich paradoxerweise, wie Claude Haas gezeigt hat, militärische Traditionalisten und radikale Kriegsgegner. Während die einen die Drohnen für den Verlust kämpferischer Tugenden verantwortlich machen, befürchten die anderen eine Entgrenzung der Gewalt, wenn automatisierte Zerstörungstechnik ihren Einsatz risikolos macht. Dass der Drohnenkrieg den Hütern soldatischer Werte suspekt ist, verwundert wenig. Wenn Pazifisten jedoch ihrer Drohnenkritik mit dem Feigheitsvorwurf Nachdruck zu verleihen suchen, geraten sie, um in der militärischen Metaphorik zu bleiben, auf vermintes Gelände: In der Geschichte des Krieges diente die Überhöhung des vermeintlich fairen Zweikampfs, als Gegenmodell zur gezielten Tötung aus sicherer Entfernung, stets dazu, „das Schlachten akzeptabel - oder besser noch, ruhmreich zu machen". ${ }^{18}$ Weil bloßer Zwang auf Dauer nicht ausreicht, um Menschen dazu zu bringen, in den Krieg zu ziehen,

13 Mark Bowden: The Killing Machines. How to Think About Drones, in: The Atlantic, September 2013.

14 F. S. Naiden: Heroes and Drones. Drones Fly in the Face of Lessons Taught to us by Centuries of Warfare, in: The Wilson Quarterly, Autumn 2013.

15 Homer: Ilias, übers. von Johann Heinrich Voß, Stuttgart 1968, 11, 386-387.

16 Vgl. Grégoire Chamayou: Ferngesteuerte Gewalt. Eine Theorie der Drohne, Wien 2014, S. 110. Die folgenden Ausführungen verdanken Chamayous Buch zahlreiche Anregungen.

17 Vgl. dazu Claude Haas: Zum Triumph des Helden in der Drohnendebatte, in: Merkur 69, Heft 793, Juni 2015, S. 70.

18 Chamayou: Ferngesteuerte Gewalt (Anm. 16), S. 108. 
andere zu töten und sich selbst in Gefahr zu bringen, getötet zu werden, weil die Staatsräson oder welche Ziele auch immer aber genau dies von ihnen verlangen, wird die Kopplung von Kampf und Opfer zur heroischen Tat verklärt. Die Fabrikation gehorsamer Soldaten muss beides wecken, die Bereitschaft zu töten und die zu sterben, und zu diesem Zwecke werden diejenigen, die zum einen wie zum anderen willens und in der Lage sind, zu Vorbildern erhoben und als Helden verehrt. Das Ethos des fairen Kampfs liefert dafür das normative Gerüst: Die Gefahr, selbst getötet zu werden, suspendiert das allgemeine Tötungsverbot. Nur weil der Gegner mir ans Leben will und kann, so das militärische Ethos, darf und muss ich ihm das seine nehmen.

Mit der kriegerischen Wirklichkeit hatten die Beschwörungen militärischen Heldentums indes niemals viel zu tun. Das Letzte, was sich Soldaten auf dem Schlachtfeld wünschen, ist ein fairer Kampf. ${ }^{19}$ Sie wollen überleben, keine Verletzungen davon tragen, nicht in Gefangenschaft geraten, vielleicht Beute machen, sich rächen, ihre Gegner außer Gefecht setzen oder einfach nur töten, und sie werden deshalb alles tun, um auf jeden Fall zu den Stärkeren zu gehören. Die Geschichte militärischer Rüstung lässt sich als ein einziger Versuch lesen, die Symmetrie der Konfrontation durch technische Überlegenheit zu asymmetrisieren, was durch immer neue Resymmetrisierungsversuche konterkariert wird, die wiederum neue Asymmetrisierungsanstrengungen in Gang setzen und so weiter. ${ }^{20} \mathrm{Im}$ Krieg kreuzen sich zwei Handlungslogiken, die des Kampfes und die der effizienten Gewaltanwendung. Auf der einen Seite ist der Krieg nach Clausewitz' berühmter Definition „nichts als ein erweiterter Zweikampf“, in dem jede Partei versucht, die andere „durch physische Gewalt zur Erfüllung [ihres] Willens zu zwingen“, sie „niederzuwerfen und dadurch zu jedem ferneren Widerstand unfähig zu machen". Auf der anderen Seite rüstet sich die Gewalt, wie Clausewitz nur wenige Zeilen später schreibt, „mit den Erfindungen der Künste und Wissenschaften aus, um der Gewalt zu begegnen “. ${ }^{21}$ Jede Seite versucht durch Einsatz technischer Mittel, die andere Seite wehrlos zu machen und sich zugleich gegen deren Gewalt wirksam zu schützen. Dazu dienen technische Apparaturen und soziotechnische Arrangements, welche die Intensität der Gewalt, ihre Zielgenauigkeit und Reichweite steigern, die Beweglichkeit und Geschwindigkeit von Truppen und Waffen erhöhen, für möglichst vollständige Sichtbarkeit des Gegners sorgen oder durch Panzerung beziehungsweise Tarnung die eigene Verwundbarkeit minimieren sollen. Eine elementare Strategie in diesem Zusammenhang ist die Vergrößerung der Distanz zum Gegner, die wiederum eine erweiterte Reichweite und verbesserte Zielgenauigkeit der eigenen Waffensysteme voraussetzt. Die Körper der Kämpfer

19 „As anyone who has ever been in combat will tell you, the last thing you want is a fair fight", Bowden: Killing Machines (Anm. 13).

20 Vgl. Herfried Münkler: Der Wandel des Krieges. Von der Symmetrie zur Asymmetrie, Weilerswist 2006.

21 Carl von Clausewitz: Vom Kriege, Bonn 161952, S. 89-90. 
und ihre Waffen, genauer: der Ort, an dem die Waffen ihre Zerstörungskraft entfalten, werden möglichst weit voneinander getrennt. Das Ziel ist es, den Gegner zu treffen, ohne selbst von ihm getroffen werden zu können. Clausewitz erkennt darin eine Entemotionalisierung des militärischen Handelns: „Die Waffen, womit der Feind schon in der Entfernung bekämpft wird, sind mehr Instrumente des Verstandes; sie lassen die Gemütskräfte und den eigentlichen Kampfinstinkt fast ganz ruhen, und zwar umso mehr, je größer die Entfernung ist, in der sie wirksam sind. Bei der Schleuder kann man sich noch einen gewissen Ingrimm denken, mit dem sie geworfen wird, weniger schon beim Büchsenschuß, noch weniger beim Kanonenschuß." ${ }^{22}$ Vermutlich zeitigt allein diese affektive Abkühlung deheroisierende Effekte: Bewunderung und Verehrung vermag eher die Leidenschaft des Kämpfers zu wecken als die Nüchternheit des Technikers.

Die Drohnenkriegführung treibt die Asymmetrie von Kampf und technischer Effizienz so weit ins Extrem, dass die eine Seite ganz verschwindet. Die Spielregeln wandeln sich radikal: „Das Paradigma ist nicht jenes von zwei Kämpfern, die einander gegenüberstehen, sondern ein anderes: ein Jäger, der seinen Vorstoß macht, und eine Beute, die flieht oder sich versteckt. ${ }^{\text {"23 }}$ Der Krieg wird zur präventiven Menschenjagd: „Es geht weniger darum, spezifische Angriffe zu erwidern, als vielmehr die Entstehung neuer Bedrohungen durch die frühzeitige Ausschaltung ihrer potenziellen Agenten zu verhindern. ${ }^{\text {" } 24}$ Drohnen machen keine Gefangenen, und sie erlauben keine Kapitulation. „That others may die“, steht auf einem emblematischen Aufnäher, mit dem die Reaper-Crews ihre Uniform zieren. ${ }^{25}$

Das Bemühen, eigene Verluste zu vermeiden, ist allerdings kein Spezifikum des Drohnenkriegs, und auch die Einseitigkeit des Tötens hat historische Vorläufer. Als die westlichen Eroberer in den Kolonialkriegen mit Maschinengewehren die allenfalls mit Speeren oder alten Flinten bewaffneten Eingeborenen niedermähten, hatte auch das nichts Heldenhaftes. Das Besondere der ,Drohnisierung' des Krieges liegt nicht in der imperialen Machtüberlegenheit, sondern im offiziellen Übergang „von einer Ethik der Aufopferung und Tapferkeit zu einer Ethik der Selbsterhaltung und mehr oder weniger akzeptierten Feigheit“. ${ }^{26}$ Für die westliche Militärpolitik wird der Schutz des Lebens der eigenen Soldaten zum absoluten Imperativ. Schon eine begrenzte Anzahl von Gefallenen - gemeint sind selbstverständlich nur Tote auf der eigenen Seite - würde die öffentliche Zustimmung zu einem Kriegseinsatz gefährden, so die militärische Begründung für die Umwertung militärischer Werte. Smarte Technologie soll deshalb übernehmen, wofür bisher Kampfeswille und Opferbereitschaft mobilisiert werden mussten. „Present circumstances“, schrieb bereits 1995 der US-amerikanische Politikwissenschaftler und Strategie-Experte

Ebd., S. 1007.

Chamayou: Ferngesteuerte Gewalt (Anm. 16), S. 44.

Ebd., S. 46.

Vgl. ebd., S. 103.

Ebd., S. 112. 
Edward N. Luttwak in einem Artikel für „Foreign Affairs“, der die Debatte um die postheroische Kriegführung eröffnete, „call for even more than a new concept of war, but for a new mentality that would inject unheroic realism into military endeavor precisely to overcome excessive timidity in employing military means. ${ }^{\text {" } 27}$ Nicht Kriegsverhinderung, sondern die Wiedergewinnung der Kriegführungsfähigkeit motiviert die Abkehr vom Ideal militärischen Heldentums. - „Give War a Chance" lautet der Titel eines anderen Aufsatzes von Luttwak aus dem Jahre 1999.28

Die normative Umstellung vollzieht sich allerdings keineswegs bruchlos. Ganz auf heroisierende Rhetorik und Rituale glaubt die US-Militäradministration auch im Tele-Krieg nicht verzichten zu können. So verkündete das Verteidigungsministerium am 13. Februar 2013 die Einführung eines Ordens für Drohnenkrieger. Die Distinguished Warfare Medal sollte in der Hierarchie der Auszeichnungen über dem Purple Heart, dem Orden für im Kampf verwundete Soldaten, rangieren. Verliehen werden sollte sie an Einsatzkräfte, deren außerordentliche Leistungen unabhängig von ihrer Distanz zum traditionellen Gefechtsfeld besondere Anerkennung verdienten. „We should also have the ability to honor extraordinary actions that make a true difference in combat operations, even if those actions are physically removed from the fight", begründete Staatssekretär Leon Panetta die Entscheidung des Pentagon..$^{29}$ Die Ankündigung löste indes sofort Widerspruch von Veteranenverbänden aus, die darauf bestanden, eine so hochrangige Auszeichnung ausschließlich für jene zu reservieren, die tatsächlich an Kampfhandlungen beteiligt waren. In ihrer Petition forderten sie: „Under no circumstance should a medal that is designed to honor a pilot [who] is controlling a drone via remote control, thousands of miles away from the theater of operation, rank above a medal that involves a soldier being in the line of fire on the ground. This is an injustice to those who have served and risked their lives and this should not be al-

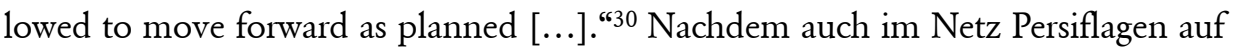
die „Nintendo Medal“ kursierten, zog das Verteidigungsministerium zwei Monate später seine Ankündigung zurück. ${ }^{31}$

In der Geschichte des Krieges führten neue und besonders wirkmächtige Waffen häufig auch zur Heroisierung derjenigen, die sie trugen oder lenkten - man

27 Edward N. Luttwak: Toward Post-Heroic Warfare, in: Foreign Affairs 74, May/June 1995, S. 122.

28 Ders.: Give War a Chance, in: Foreign Affairs 78, July/August 1999, S. 36-44.

29 „The Distinguished Warfare Medal will be awarded in the name of the secretary of defense to service members whose extraordinary achievements, regardless of their distance to the traditional combat theater, deserve distinct department-wide recognition“, Presseerklärung des US-Department of Defense, Release No 084-13, 13. Feburar 2013, http://www.defense. gov/releases/release.aspx?releaseid=15817, 2. Mai 2015. 
denke nur an die Fliegerhelden des Ersten und Zweiten Weltkriegs. Für die Drohnenpiloten trifft das Gegenteil zu: Sie sehen sich dem Vorwurf ausgesetzt, unverbesserliche Nerds zu sein, die ihrer puerilen Leidenschaft für Computerspiele nachgehen und vom sicheren Sessel aus die Raketen schon deshalb ohne Skrupel abfeuern, da sie zwischen virtueller und realer Welt kaum mehr zu unterscheiden wüssten. Der Gamifizierung des Krieges entspreche eine Playstation-Mentalität der "chair-borne rangers“, die ihre prospektiven Opfer nur als bewegte Bilder auf den Monitoren sehen. Die Air Force klagt über ein „drone stigma“, dem die Crews ausgesetzt seien, und hat Mühe, ausreichend qualifiziertes Personal zu finden: „Most pilots don’t enjoy flying from a box." ${ }^{\text {"32 }}$

Nachdem in der Anfangsphase der Drohnenangriffe wiederholt Interviewäußerungen von Piloten bekannt wurden, die geeignet waren, den Eindruck zu bestätigen, hier fänden Computerspieljunkies ein ideales Betätigungsfeld, betonen die militärischen Instanzen inzwischen die besonderen psychischen Belastungen, denen die Drohnen-Operatoren ausgesetzt sein sollen. Die permanente Sorge, versehentlich Unschuldige zu treffen sowie das emotionale Wechselbad, in der Nachtschicht per Fernsteuerung verdächtige Terrorkämpfer zu töten und am nächsten Morgen die Kinder zur Schule zu bringen, stellen demnach außergewöhnliche Stressoren dar und erhöhen das Burnout-Risiko.

Die Befunde in der militärmedizinischen Fachliteratur sehen freilich anders aus: Die untersuchten Operatoren wiesen zwar deutlich überdurchschnittliche BurnoutRaten auf, die Befragten nannten als Belastungsfaktoren jedoch in erster Linie Schichtarbeit, Dienstplanänderungen, personelle Unterbesetzung und vor allem die Eintönigkeit der Arbeit, wie der Militärspychiater Hernando Ortega ausführt:

„It's really kind of a boring job to be vigilant on the same thing for days and days and days. It's really boring. It's kind of terrible. And maintaining relationships with their families - these were the kinds of things that they reported as that were stressful for them. And if you look through that stuff, they don't say because I was in combat. They don't say because we had to blow up a building. They don't say because we saw people get blown up. That's not what causes their stress - at least subjectively to them. It's all the other quality of life things that everybody else would complain about too."33

Heldenmythen lassen sich aus solchen Äußerungen schwerlich stricken.

32 Lee Ferran: Drone 'Stigma' Means 'Less Skilled' Pilots at Controls of Deadly Robots, in: ABC News, 29. April 2014, http://abcnews.go.com/Blotter/drone-stigma-means-skilledpilots-controls-deadly-robots/story?id=23475968, 2. Mai 2015.

33 Hernando J. Ortega: Combat Stress in Remotely Piloted/UAS Operations, Transkript eines Vortrags im Brookings-Institut, Washington, 3. Februer 2012, S. 24, http://www.brookings. edu/events/2012/02/03-military-medical-issues, 2. Februar 2015. Vgl. mit ähnlichen Befunden auch Joseph A. Ouma [et al.]: Facets of Occupational Burnout Among US Air Force Active Duty and National Guard/Reserve MQ-1 Predator and MQ-9 Reaper Operators, in: Final Report for July 2010 to June 2011 of the Air Force Research Laboratory, AFRL-SA-WP-TR-2011-0003, June 2011, http://www.dtic.mil/cgi-bin/GetTRDoc?AD=AD A548103, 2. Mai 2015. 
Wenn also die Drohnenkrieger schon nicht als Kriegshelden taugen, lassen sich dann vielleicht die Drohnen selbst heroisch aufladen? Gewissermaßen vom Objekt des Heroischen zum heroisierten Objekt. An entsprechender Rhetorik mangelt es nicht: Die militärische Propaganda rühmt die elektronischen Aufklärungs- und Waffensysteme dafür, das Leben der eigenen Truppen zu schützen und durch ihre Präzision auch die Zahl der gegnerischen Opfer zu senken. „Im Vergleich zu Jagdbombern sind Drohnen Systeme, die im Prinzip die Gewaltintensität minimieren“, schreibt der Politikwissenschaftler Herfried Münkler. „Es geht nicht ohne Blutvergießen ab. Aber die Höhe der Kollateralschäden ist in den vergangenen zwei, drei

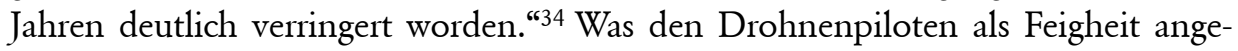
kreidet wird, die Ausschaltung des Gegners ohne Risiko, wird der Technik als moralische Qualität gut geschrieben. Ihren Apologeten gelten die Drohnen als geradezu humanitäre Waffen, die zuverlässig jene Jobs erledigen, die „dull, dirty and dangerous“ ", 35 stumpfsinnig, dreckig und gefährlich sind. Sie spüren die Bösen auf, und angeblich nur diese, und vernichten sie, noch bevor sie zur Untat schreiten können. All das sind militärische Leistungen, für die Soldaten zweifellos in den Heldenstand erhoben würden. Dass Drohnen weit länger auf ihrem Posten in der Luft bleiben, schärfer sehen und genauer treffen, als es Menschen je könnten, ist ohnehin klar.

Bedeutet Postheroismus also die Delegation heldenhafter Tugenden an Maschinen, die möglicherweise bald auch auf die menschliche Fernsteuerung verzichten werden? In den Hightech-Waffenschmieden experimentiert man jedenfalls bereits eifrig mit vollautomatisierten Systemen. Münkler träumt sogar schon von einem Krieg ganz ohne Opfer: „Man kann sich vorstellen, dass Kriege irgendwann nicht mehr letal ausgetragen werden, sodass dabei keine Menschen mehr zu Schaden kommen, sondern die Fähigkeiten einer Seite werden durch die andere Seite aufgrund überlegener Technologie ausgeschaltet und die Unterlegenen kapitulieren, sprich resignieren in ihrem politischen Willen. "36 Die Drohne erscheint in solchen apologetischen Phantasmen einer Kriegführung ohne tötende Gewalt gleichermaßen als technisches Substitut wie als geradezu hegelianische Aufhebung militärischen Heldentums. Der „prometheischen Scham“, dem unhintergehbaren Inferioritätsgefühl der Menschen angesichts der Überlegenheit der von ihnen geschaffenen technischen Werkzeuge, das der Philosoph Günther Anders den Menschen des Atomzeitalters attestierte, ${ }^{37}$ korrespondiert die ehrfürchtige Bewunderung ebendieser Werkzeuge.

34 Matthias Schiermeyer: Technische Sprünge verändern die Kriegsführung. Interview mit Herfried Münkler, in: Stuttgarter Zeitung, 17. Juli 2014.

36 Ebd.

37 Günther Anders: Über prometheische Scham, in: Die Antiquiertheit des Menschen, Bd. 1, Über die Seele im Zeitalter der zweiten industriellen Revolution, München ${ }^{6} 1983$, S. 21-95. 
Von einem Heldenkult rund um die Drohnen kann trotzdem keine Rede sein. Dazu fehlen den Unmanned Combat Air Vehicles jene spezifisch menschlichen Eigenschaften - allen voran moralische Urteilskraft, Empathie und Emotionalität -, an die heroische Identifikationen anschließen können. In den Imaginationswelten der Populärkultur wimmelt es zwar von anthropomorphisierten Robotern, die aber nur dann zu Helden avancieren, wenn sie menschliche Regungen zeigen, also ihre Roboterhaftigkeit im entscheidenden Moment aufgeben. Maschinen selbst operieren nicht im Heldenmodus, ihnen fehlt dafür eine fundamentale Dimension von Handlungsmacht: die Fähigkeit, sich zu entscheiden. Sie prozessieren Algorithmen; heroischen Anrufungen zu folgen oder eben nicht, dafür besitzen sie kein Sensorium.

Helden erzeugen die Drohnen allerdings auf ganze andere Weise: Das ferngesteuerte ,targeted killing führt dem globalisierten Dschihadismus fortlaufend neue Kämpfer zu. Sie setzen der Risikoaversion westlicher Kriegführung die Unbedingtheit ihres Todeswillens entgegen und finden dafür begeisterte Anhänger. Der suicide bomber ist die feindliche Komplementärfigur des Drohnenpiloten. „Auf der einen Seite das vollkommene Engagement, auf der anderen die absolute Distanzierung.“ Während im Selbstmordattentat „der Körper des Kämpfers vollständig mit seiner Waffe verschmilzt, garantiert die Drohne die radikale Trennung der beiden. ${ }^{\text {“38 Der }}$ postheroische Traum einer sauberen Kriegführung gebiert heroische Ungeheuer.

Die Diagnose des postheroischen Zeitalters bedeutet daher keinesfalls ein Ende heroischer Anrufungen. Solange politische oder religiöse Mächte auf die Bereitschaft zum Selbstopfer angewiesen sind und sie schüren, wird man Helden suchen und finden. Der Streit darüber, ob militärischer Heroismus antiquiert ist, und wir in der Ära des Postheroismus angekommen sind, führt deshalb nicht weiter. Schon die Frage ist falsch gestellt. In Abwandlung des bekannten Buchtitels von Bruno Latour $^{39}$ müsste man stattdessen konstatieren: Wir sind nie heroisch gewesen. Wir sollten es immer nur sein. Und viel zu oft wollten wir es auch.

\section{Abbildungsnacbrweis}

Abb. 1: commons.wikimedia.org.

38 Chamayou: Ferngesteuerte Gewalt (Anm. 16), S. 95-96.

39 Bruno Latour: Wir sind nie modern gewesen. Versuch einer symmetrischen Anthropologie, Frankfurt am Main 1998. 
